\title{
Congress Calendar
}

\section{Respirition}

\begin{tabular}{l}
\hline 2.6.-5.6.2010 \\
Prague \\
Czech Republic \\
\hline 3.6.-6.6.2010 \\
Plovdiv \\
Bulgaria \\
\hline 5.6.-9.6.2010 \\
London \\
UK \\
\hline
\end{tabular}

\section{6.-12.6.2010}

Bucharest

Romania

\section{6.-13.6.2010}

Samos

Greece

\begin{tabular}{l}
\hline 13.6.-16.6.2010 \\
Budapest \\
Hungary \\
\hline 19.6.-21.6.2010 \\
Vienna \\
Austria \\
\hline 5.7.-9.7.2010 \\
Ancona \\
Italy \\
\hline
\end{tabular}

18.9.-22.9.2010

Barcelona

Spain

\subsection{0.-9.10.2010}

Graz

Austria

\subsection{0.-15.10.2010}

Ancona

Italy
Pulmonary Circulation 2010 European Forum

Bulgarian Respiratory Society Third Congress

XXIX Congress of the European Academy of Allergology and Clinical Immunology

21st Congress of Romanian Society of Pneumology

International Postgraduate Symposium on Lung Cancer

16th World Congress for Bronchology

16th World Congress for Bronchoesophagology

ICPP IX / 9th International Congress on Pediatric Pulmonology

Weeks in Interventional Pulmonolgy

Flexible and Rigid Bronchoscopy

ERS 20th Annual Congress

Jahrestagung 2010 der Österreichischen Gesellschaft für Pneumologie (ÖGP)

Weeks in Interventional Pulmonolgy Flexible and Rigid Bronchoscopy

ESTS School of Thoracic Surgery Minimally Invasive Lung Surgery and Associated Treatments
Information: http://www.pc2010.eu/en/welcome

Information: http://www.thorax-bg.com

Information: Congrex Sweden AB, Stockholm

E-Mail eaaci2010@congrex.com

www.congrex.com

\section{Information:}

90 Viilor Street, Sector 5

RO-050159 Bucharest (Romania)

Tel./Fax +40 213374460

E-Mail office@srp.ro

www.srp.ro

Information: Forum Travel \& Congress

24 Mitropoleos str.

GR-54624 Thessaloniki (Greece)

Tel. +30 231025 7128, Fax +302310231849

E-Mail info@forumcongress.com

www.congressforum.com/6lungcancer

\section{Information:}

www.wcbwcbe2010.org

Information: http://www.cipp-meeting.com

Information: AIPO Ricerche

Via Antonio da Recanate, 2

IT-20124 Milano (Italy)

Tel. +3902 3659 0354, Fax +3902 36590360

E-Mail s.gasparini@fastnet.it, www.eabip.org

Information: ERS Headquarters, 4, Avenue Ste. Luce

CH-1003 Lausanne (Switzerland)

Tel. +4121213 0101, Fax +41212130100

E-Mail info@ersnet.org,www.ersnet.org

Information: Ärztezentrale Med. Info

Helferstorferstraße 4, AT-1014 Wien

Tel. +43 1531 1638, Fax +43 15311661

E-Mail azmedinfo@media.co.at

www.ogp.at

\section{Information: AIPO Ricerche}

Via Antonio da Recanate, 2

IT-20124 Milano (Italy)

Tel. +39023659 0354, Fax +390236590360

E-Mail s.gasparini@fastnet.it, www.eabip.org

Information: http://www.estsschool.org 


\begin{tabular}{l}
\hline 20.10.-24.10.2010 \\
Antalya \\
Turkey \\
\hline 20.10.2010 \\
Antalya \\
Turkey \\
\hline
\end{tabular}

30.10.-4.11.2010

Vancouver, B.C.

Canada

16.11.-19.11.2010

Oxford

UK

22.11.-25.11.2010

Manila

Philippines

\subsection{2.-5.12.2010}

Budapest

Hungary

10.3.-12.3.2011
Marseille
France

13.5.-18.5.2010

Denver, Colo.

USA

15.6.-18.6.2011

Maastricht

The Netherlands 32nd Annual Congress of the Turkish Respiratory Society

Interventional Bronchoscopy Course: From Diagnosis

to Treatment with the Cooperation of the Turkish

Respiratory Society and the European Association for Bronchology \& Interventional Pulmonology

\section{CHEST 2010}

Future CHEST Meeting

CHEST 2011 - 22.10.-27.10.2011, Honolulu, Hawaii, USA

The Oxford/Marseille Interventional Thoracic Course

15th Annual Congress of Asian Pacific Society of Respirology

12th Central European Lung Cancer Conference (CELCC)

1st European Congress for Bronchology and Interventional Pulmonology

Presented by the European Association for Bronchology and Interventional Pulmonology

ATS 2011 - American Thoracic Society

WASOGBAL 2011
Information:

www.solunum.org.tr/solunum2010

Information:

www.solunum.org.tr/solunum 2010

www.eabip.org

Information: ACCP Member Services

3300 Dundee Road, Northbrook, IL 60062 (USA)

Tel. +1 847498 1400, Fax +1 8474985460

E-Mail registrationschestnet.org, www.chestnet.org

\section{Information:}

E-Mail david.breen@orh.nhs.uk

www.bronchoscopy-marseille.com

Information: Philippine College of Chest Physicians

Tel. +632924 9204, Fax +6329240144

E-Mail pccp@zpdee.net

www.apsr2010.org

Information: http://www.xn--celcc2010budapest-i12j.hu

Information: Atout Organisation Science

Tel. +33496151250

Fax +33496152151

E-Mail ecbip2011@atout-org.com,www.eabip.com

Information: www.thoracic.org

Information: WASOGBAL 2011 Foundation

Maastricht Unversity Medical Centre

E-Mail ild.care@mumc.nl

www.wasogbal2011.nl 